\title{
ON MODULES OF TRIVIAL COHOMOLOGY OVER A FINITE GROUP, II (FINITELY GENERATED MODULES)
}

\author{
TADASI NAKAYAMA
}

Let $G$ be a finite group. $A$ (left) $G$-module $A$ of $G$ is said to be of trivial cohomology when $H^{n}(H, A)=0$ for all rational integers $n$ and for all subgroups $H$ of $G$. The main purpose of the present note is to determine the structure of finitely generated $G$-modules of trivial cohomology, which turns out to be remarkably simple (See Theorem 1 and Corollary 3 below). We prove also an (easy) localization theorem for cohomological triviality.

However, first we recall a structural study of modules of trivial cohomology made in Part I (Illinois Math. Journ. 1 (1957), p. 36). It begins with considering a free $G$-module $A_{0}$ of which a given $G$-module $A$ is a $G$-homomorphic image. Let $A_{1}$ be the kernel of the homomorphism. Then the $G$-module $A$ is of trivial cohomology if and only if the $G$-module $A_{i}$ is so. Fiacing thus reduced the problem to the cas? of $a(Z$-)torsion-free (even $Z$-free) $G$-module, we have, as we have shown in I,

Proposition 0. A $(Z$-)torsion-free $G$-module $A$ is of trivial cohomology, if and only if for each prime $p$ (dividing the order $[G]$ of $G$ ) the residue-module $A / p A$ is $Z(p)\left[H_{p}\right]$-free, where $Z(p)\left[H_{p}\right]$ denotes the group algebra of a $p$-Sylow subgroup $H_{p}$ of $G$ over the field $Z(p)$ of rational integers $\bmod p$.

Here " $Z(p)\left[H_{p}\right]$-free" may be replaced by " $Z(p)\left[H_{p}\right]$-projective" since $Z(p)\left[H_{p}\right]$ is primary. Moreover

Proposition $0^{\prime}$. The condition in Proposition 0 may be replaced by that for every prime $p$ (dividing $[G]$ ) $A: p A$ is $Z(p)[G]$-projective.

Indeed, a $Z(p)[G]$-module is $Z(p)[G]$-projective if and only if it is $Z(p)\left[H_{p}\right]$ projective. For, since the index $\left[G: H_{p}\right]$ is inversible in $Z(p)$, any $Z(p)[G]$ module $B$ is relatively projective with respect to the subring $Z(p)\left[H_{p}\right]$, (or, what

Received April 10, 1957. 
amounts to the same, $B$ is a $Z(p)[G]$-direct summand of the $Z(p)[G]$-module $B^{*}=Z(p)[G] \otimes \otimes_{Z(p)\left[H_{l}\right]} B \approx Z\left[\left(G / H_{p}\right)_{L}\right] \otimes_{Z(p ;} B$ induced by $B$ considered as $Z(p)\left[H_{p}\right]$-module, where on the left hand side of the isomorphism sign the operation of $G$ is explained by the multiplication on $Z(p)[G]$ from left while in the right hand side the operation of $G$ is explained by the operation on both factors $Z\left[\left(G / H_{p}\right)_{L}\right], B$ and the first factor $Z\left[\left(G / H_{D}\right)_{L}\right]$ denotes the vector space over $Z(p)$ spanned by the left cosets of $H_{p}$ in $G$; the isomorphism is given by associating $\sigma_{i} \otimes_{Z(p)\left[H_{1},\right]} b$ in the left hand side to $\sigma_{i} H_{p} \otimes_{Z(p)} \sigma_{i} b$ in the right hand side, where $\left\{\sigma_{i}\right\}$ is a representative system of the left cosets of $H_{p}$ in $G$ ). Indeed, if $C$ is a $Z(p)[G]$-module having $Z(p)[G]$-submodule $D$ such that there is a $Z(p)\left[H_{p}\right]$-submodule $M$ with $C=D+M$ (direct), then, on denoting by $\pi$ the projection of $C$ onto $M$ with respect to this direct decomposition, we have a direct decomposition $C=D+\rho C$ into $Z(p)[G]$-modules (indeed $D=(1-\rho) C$ ) by putting $\rho=\left[G: H_{p}\right]^{-1} \sum \sigma_{i}^{-1} \pi \sigma_{i}$, where $\left\{\sigma_{i}\right\}$ is as above (cf. [3], [4]).

\section{Finitely generated modules of trivial cohomology}

Theorem 1. A finitely generated (Z-)torsion-free G-module $A$ is of trivial cohomology if and only if $A$ is a direct summand of a free G-module, or, what is the same, if and only if $A$ is $Z[G]$-projective, where $Z[G]$ is the group algebra of $G$ over the ring $Z$ of rational integers.

As the "if" part is evident, we prove the "only if" part. Let, to do so, $A$ be a finitely generated torsion-free G-module of trivial cohomology. Let $p$ be any rational prime and $H_{p}$ be a $p$-Sylow subgroup of $G$. By Proposition 0 the residue-module $A / p A$ has an independent basis over $Z(p)\left[H_{p}\right]$. Let $a_{1}, \ldots, a_{n}$ be representatives in $A$ of the basic elements. Denote, further, the quotient ring of $Z$ with respect to $p$ by $Z_{p}$. As $A$ is $Z$-free, the tensor product $A_{p}=A \otimes_{z} Z_{p}$ is $Z_{p}$-free and $A$ may be looked upon as a $G$-submodule of $A_{p}$. We contend that $a_{1}, \ldots, a_{n}$ form an independent $Z_{p}\left[H_{p}\right]$-basis of $A_{p}$. Indeed, since $A_{p} / p A_{p}$ is naturally isomorphic with $A / p A$, the residue-classes of $a_{1}, \ldots, a_{n}$ modulo $p A_{p}$ form an independent $Z(p)\left[H_{p}\right]$-basis of $A_{p} / p A_{p}$, or, what amounts to the same, the $\left[H_{p}\right] n$ elements $\alpha a_{i} \bmod p A_{p}, \alpha$ running over $H_{p}$, form an independent $Z(p)$-basis of $A_{p} / p A_{p}$. It follows then readily that the matrix of transformation from an independent $Z$-basis of $A$ to our $\left[H_{p}\right]^{*} n$ elements $\alpha a_{i}$ has a determinant prime to $p$, whence inversible in $Z_{p}$. This shows that $\alpha a_{i}$ form an independent 
$Z_{p}$-basis of $A_{p}$, or equivalently, $a_{i}$ form an independent $Z_{p}\left[H_{p}\right]$-basis of $A_{b}$.

Now, since $\left[G: H_{p}\right]$ is inversible in $Z_{p}$, every $Z_{p}[G]$-module is relatively projective with respect to $Z_{p}\left[H_{p}\right]$; the proof is the same as was made above in context of Proposition $0^{\prime}$. As our $A_{p}$ has been seen to be $Z_{p}\left[H_{p}\right]$-free, this implies that $A_{p}$ is $Z_{p}[G]$-projective. Since this is the case for every rational prime $p$, Theorem 1 now follows from the following lemma which is of interest and significance by itself.

Lemma 2. A finitely generated $G$-module $A$ is $Z[G]$-projective if, and only if, for every rational prime $p$ the tensor product $A_{p}=A \otimes_{Z} Z_{p}$ is $Z_{p}[G]$-projective, where $Z_{p}$ is the ring of quotients of $Z$ with respect to $p$. (More precisely, we have $\left.\operatorname{dim}_{Z[G]} A=\sup _{p} \operatorname{dim}_{Z_{p}[G]} A_{p}\right)$.

This lemma may be proved as follows by an argument of Serre [6]; cf. also [1], VII, Exer. 11 (Observe, however, that the lemma itself is not contained in $[6]$, nor in $[1])$. Let, thus,

$$
0 \leftarrow A \leftarrow F_{0} \leftarrow F_{1} \leftarrow \ldots
$$

(exact)

be a resolution of $A$ consisting of finitely generated free $G$-modules $F_{i}$. Setting $\left(F_{i}\right)_{p}=F_{i} \otimes_{z} Z_{p}$ we obtain a resolution

$$
0 \leftarrow A_{p} \leftarrow\left(F_{0}\right)_{p} \leftarrow\left(F_{1}\right)_{p} \leftarrow \ldots
$$

of $A_{p}$ by $Z_{p}[G]$-free modules $\left(F_{i}\right)_{p}$. As each $F_{i}$ has an independent finite basis over $Z[G]$, we see, for any $G$-module $C$, that the $Z[G]$-module $\operatorname{Hom}_{Z[i]}\left(F_{l}, C\right)$ is simply a direct sum of a finite number of copies of $C$ and hence the $Z_{p}[G]$-module $\left(\operatorname{Hom}_{Z[G]}\left(F_{i}, C\right)\right)_{p}=\operatorname{Hom}_{Z[G j}\left(F_{i}, C\right) \otimes_{z} Z_{p}$ is isomorphic to $\operatorname{Hom}_{Z_{i}[G]}\left(\left(F_{i}\right)_{p}, C_{p}\right)$ (which is the direct sum of the same finite number of copies of $\left.C_{p}\right), \quad C_{p}$ being $C \otimes_{z} Z_{p}$. We have then readily $\operatorname{Ext}_{\angle[i]}^{t}(A, C)$ iर $_{z} Z_{p}$ $\approx \operatorname{Ext}_{Z_{i}[(i]}^{i}\left(A_{p}, C_{p}\right)$. Now, if $A_{p}$ is $Z_{p}[G]$-projective, for every $p$, then the right hand side vanishes for $i>0$, and the same must be the case case for the left hand side. $\operatorname{Ext}_{Z[G]}^{i}(A, C)$ being finitely generated in case $C$ is so, this implies $\operatorname{Ext}_{Z\lceil G]}^{i}(A, C)=0$ for $i>0$ whenever $C$ is finitely generated. It follows that $A$ must be $Z[G]$-projective. The converse is rather evident.

Theorem 1 being thus proved, we may apply it to the kernel of an epimorphism of a free $G$-module to a given module, to obtain:

Corollary 3. A finitely generated G-module is of trivial cohomology if and 
only if it is a residue-module of a finitely generated free G-module modulo a $Z[G]$-projective submodule.

Each of the following two propositions, in which $Z_{p}$ denotes as above the ring of quotients of $Z$ with respect to $p$, can readily be seen from a portion of our proof to Theorem 1:

Proposition 4. Let $A$ be a $Z_{p^{-}}$(or $Z_{p}[G]$-) finitely generated $\left(Z\right.$ - or $Z_{p^{-}}$) torsion-free $Z_{p}[G]$-module ( the operation of the elements of $Z_{p}$ being commutative with the operation of the elements of $G$ ). Each of the following conditions i), ii), iii) is necessary and sufficient for $A$ to be of trivial cohomology: i) $A$ is $Z_{p}[G]$-projective; ii) $A$ is $Z_{p}\left[H_{p}\right]$-projective (where $H_{\dot{\nu}}$ is a $p$-Sylow subgroup of $G)$; iii) $A$ is $Z_{p}\left[H_{p}\right]$-free.

(Assume that ii) is the case. Then $A$ is of trivial cohomology and hence satisfies iii), as well as i), by our proof to Theorem 1.)

Proposition 5. Let $A$ be a $(Z$-, or $Z[G]$-) finitely generated $(Z-)$ torsion free $G$-module. $A$ is of trivial cohomology if and only if $A_{p}=A \otimes_{z} Z_{p}$ is $Z_{p}[G]$ projective for every prime $p$ (dividing $[G]$ ). Alternative ways of stating the condition can be seen from Proposition 4.

The following proposition may be of interest in view of the (probably) open question whether every finitely generated $Z[G]$-projective module is $Z[G]$ free (cf. [1], p. 241):

Proposition 6 . Let $A$ be a finitely generated $Z[G]$-projective module. Then the $Z$-rank of $A$ is a multiple of the order $[G]$.

For, with any prime $p, A / p A$ is $Z(p)\left[H_{p}\right]$-free. Hence the $Z(p)$-rank of $A / p A$ is a multiple of $\left[H_{p}\right]$. But the $Z$-rank of $A$ is clearly equal to the $Z(p)$ rank of $A / p A$. Thus the $Z$-rank of $A$ is a multiple of $\left[H_{p}\right]$. Since this is the case for every $p$, we have the assertion.

\section{A localization theorem}

Propositions $0,0^{\prime}$ and 5 have evidently the effect of localization with respect to the property of cohomological triviality, while Lemma 2 is naturally a localization lemma for projectivity (or projective dimension in general). In stating local properties also in terms of cohomological triviality, in connection of Propositions $0,0^{\prime}$, we have 
Proposition $0^{\prime \prime}$. A torsion free $G$-module $A$ is of trivial cohomology if and only if the $G$-module $A / p A$ is of trivial cohomology for every prime $p$ (dividing $[G])$.

(This is, however, merely an easy and rather trivial portion of the content of Proposition 0 and the main feature of the latter lies in that its structural local condition is implied by the present local condition.)

Contrary to that these Propositions $0,0^{\prime}, 0^{\prime \prime}$ and 5 are for torsion-free modules only (though they have, except Proposition $0^{\prime \prime}$, merits to be structural), the following localization theorem is for general modules:

Theorem 7. A $G$-module $A$ is of trivial cohomology if and only if $A_{p}=A \otimes_{Z} Z_{p}$ is of trivial cohomology for every prime $p$ (dividing $[G]$ ), where $Z_{p}$ is the ring of quotients of $Z$ with respect to $p$.

To prove this, we construct a free $G$-module $A_{0}$ of which the given $G$ module $A$ is a $G$-homomorphic image and denote the kernel of the homomorphism by $A_{1}$. As $Z_{p}$ is $\left(Z\right.$-) torsion-free, we have $\operatorname{Tor}_{1}^{Z}\left(A, Z_{p}\right)=0$ and, therefore $0 \rightarrow A_{1} \otimes{ }_{Z} Z_{p} \rightarrow A_{0} \otimes Z_{Z} Z_{p} \rightarrow A \otimes{ }_{Z} Z_{p} \rightarrow 0$ (exact), for any prime $p$. So, for every $p$, the cohomological triviality of $A \otimes_{2} Z_{p}$ is equivalent to that of $A_{1} \otimes_{z} Z_{p}$. Since $\left(A_{1} \otimes_{z} Z_{p}\right) / p\left(A_{1} \otimes_{z} Z_{p}\right) \approx A_{1} / p A_{1}$ and $\left(A_{1} \otimes Z_{p}\right) / q\left(A_{1} \otimes_{z} Z_{p}\right)=0$ for $(q, p)$ $=1$, the $G$-module $A_{1} \otimes_{z} Z_{p}$ is of trivial cohomology if and only if $A_{1} / p A_{1}$ is so, by Proposition $0^{\prime \prime}$. But, that this is the case for every $p$ (dividing $[G]$ ) is equivalent, again by Proposition $0^{\prime \prime}$, to that $A_{1}$ is of trivial cohomology, which is in turn equivalent to that $A$ is so. (Of course we could use either of Proposition $0,0^{\prime}$ instead of Proposition $0^{\prime \prime}$.)

Remark. In Propositions 4,5 and Theorem 7 (as well as in Lemma 2) we could replace $Z_{p}$ by the ring of rational $p$-adic integers.

Remark. In the present note we have used only a small portion of Part I. Indeed, since we do not need to make dimension shifting in proving Proposition 0 (as well as Propositions $0^{\prime}, 0^{\prime \prime}$ ) the dimension shifting portion of our proof in Part I could be eliminated for our present purpose. Thus, what we have made use of, beyond the reduction (to torsion free modules and) to modules $B$ with $p B=0$, is Lemma 8 in Part $\mathrm{I}$ in which the $Z(p)[G]$-free structure is derived from $H^{-1}(G, B)=0(p B=0)$ for a $p$-group $G$. As an alternative, we shall here derive the same structure from $H^{-2}(G, G)=0(p B=0), G$ being a $p$-group. 
Indeed, since $p B=0$ we have $H^{-2}(G, B)$ (not only $=\operatorname{Tor}_{1}^{Z}(Z, B)$ but) $=\operatorname{Tor}_{1}^{Z(p)}(Z(p), B)$; this can readily be seen either directly by reducing the standard complex, say, modulo $p$ or by a change of rings formula ([1], VI, 4.1.1). As $Z(p)[G]$ is primary, $G$ being a $p$-group, $\operatorname{Tor}_{1}^{Z(p)}(Z(p), B)=0$ implies, by a syzygy theorem ([2]), that $B$ is $Z(p)[G]$-projective and, therefore, has the desired $Z(p)[G]$-free structure $([5])$.

Added in proofs: Another way of formulating Corollary 3 is, as S. Eilenberg points out, to say that a finitely generated $G$-module $A$ is of trivial cohomology if and only if $1 \cdot \operatorname{dim}_{z[\theta]} A \leqq 1$, and the same holds with the last condition replaced by $1 \cdot \operatorname{dim}_{Z[G]} A \leqq \infty$. He also points out that in proving Lemma 2 we had better to make explicit the natural isomorphism $\operatorname{Ext}_{\Lambda}^{i}(A, C)$ $\left(\Gamma \approx \operatorname{Ext}_{\Lambda \otimes \Gamma}^{i}(A \otimes \Gamma, C \otimes \Gamma)\left(\otimes\right.\right.$ standing for $\left.\otimes_{K}\right)$ for a left Noetherian $K$ algebra $\Lambda$, a $K$-flat $K$-algebra $\Gamma$, a finitely generated (left) $\Lambda$-module $A$ and a (left) $A$-module $C$.

\section{REFERENCES}

[1] H. Cartan-S. Eilenberg, Homological Algebra, Princeton 1956.

[2] S. Eilenberg, Homological dimension and syzygies, Ann. Math., 64 (1956), 328-336.

[3] Higman, Modules with a group of operators, Duke Math. Jour., 21 (1954), 369-376.

[4] F. Kasch, Grundlagen einer Theorie der Frobeniuserweiterungen, Math. Ann., 127 (1954), 453-474.

[5] H. Nagao-T. Nakayama, On the structure of $\left(M_{0}\right)$ - and $\left(M_{u}\right)$-modules, Math. Zeits, 59 (1953), 164-170.

[6] J.-P. Serre, Sur la dimension homologique des anneaux et des modules Noethériens, Proc. Intern. Symposium, Tokyo 1955.

Nagoya University 